\title{
The Quality Test of Wire Bonding
}

\author{
Caiyuan Wang \\ School of Mechanical \& Electronic Engineering \\ Tianjin Polytechnic University \\ Tianjin 300160, China \\ E-mail: 1lwinnie@126.com.cn \\ Ronglu Sun \\ School of Mechanical \& Electronic Engineering \\ Advanced Mechatronics Equipment Technology Tianjin Area Major Laboratory \\ Tianjin 300160, China \\ E-mail: rlsun@tjpu.edu.cn
}

\begin{abstract}
The reliability of the IC chip during performance of its function in any application is very much dependant on the quality of the wire bond interconnection. If the quality of the wire bond interconnection is poor and not consistent, it has a significant impact on the reliability and dependability of the device. The quality of a wire bond is determined by the strength of the interfaces between the bond wire and the bond pad as well as the bond wire and the lead finger. In this article, we discuss in detail established methodologies such as bond pull test, bond shear test, visual inspection and some special test of quality.
\end{abstract}

Keywords: Wire bonding, Quality test, Failure modes

\section{Introduction}

Today, most of the critical applications like high performance computers and industrial systems are controlled by microchips which have wire bond interconnections. The reliability of the IC chip during performance of its function in any application, be it in an automobile, space shuttle or a personal computer, is very much dependant on the quality of the wire bond interconnection. If the quality of the wire bond interconnection is poor and not consistent, it has a significant impact not only on the reliability and dependability of the device, but also has a huge economic impact. Therefore, quality assurance of the wire bond is of utmost importance in the IC assembly work flow.

\section{The Bond Pull Test}

The bond pull test is the most widely used technique for the evaluation and control of wire bond quality. This technique is also used for wire bonding process control and process optimization during assembly manufacturing. The bond pull test involves placing a hook under the wire and applying a normal upward force and the wire is pulled till it fails, as is shown in figure1.

During bond pull test, if a ball lift failure or weld lift failure occurs even at very high pull value, still it is unacceptable and the bond is considered of poor bond quality. In case of such failures the cause of the non-stick must be analyzed thoroughly and necessary remedial action needs to be taken. Examples of ball lift and weld lift failures are shown in figure2.

\subsection{Different Modes of Pull Test Failures and their Interpretation}

The major failure modes that one observes during a gold ball bond pull test are, ball lift (A type),ball neck break (B type), mid span break (C type), heel break (D type) and weld lift (E type), as shown in figure3. In special cases, the failure may also occur at other interfaces such as lift off of bond pad metallization, caterings under the bond pad and peel off of lead finger metallization. Let us review and discuss the five types of failure modes in detail.

\subsection{Ball Lift Failure}

The occurrence of ball lift failure indicates that the ball to bond pad interface is very weak. The ball lift failure indicates either the bond has not formed or the bond has become weak. Ball lift can be due to a variety of factors. Poor wire 
bonder set-up and bond pad surface contamination are primary causes of ball lifting. Poor set-up includes improper parameter settings, unstable work piece holders, and worn-out tools. These result in poor initial welding and inadequate intermetallic formation between the bond pad and the ball.

\subsection{Ball Neck Failure}

The failure of the wire just above the ball neck is shown in figure4. This is the predominant failure mode that we observe during bond pull test. Neck breaking is the severing of the wire from its ball bond due to a fracture in the neck. The neck is the portion where the wire meets the ball bond. Neck breaking is commonly due to poor wire bonder set-up such as improper bonding parameter settings, boneheads movement settings, and worn-out or contaminated tools. Incorrect bonding parameters can deform the bond excessively, resulting in a thin, weak, or cracked neck which can easily fracture. Crack propagation can also be caused by defects in the wires due to worn-out and contaminated tools.

\subsection{Wire Break at Mid Span}

Bond wire failure at the midspan is shown in figure4. This failure mode indicates that the wire failed by plastic deformation and ductile fracture, rather than by brittle fracture at the heel or at the ball neck. Usually the failure at mid span gives the highest bond pull value, close to the ultimate tensile strength (UTS) of the wire.

\subsection{Heel Break}

Heel breaking is the severing of the wire from its wedge or crescent bond due to a fracture in the heel. The heel is the portion where the wire tapers off into the bond. When we perform bond pull test, the heel break failure may occur as shown in figure4 and this usually indicates damaged heel area. Improper bonehead's movements and low loop settings may subject the wires to excessive stresses that tend to pull them backward and away from the bonds, resulting in gross heel cracks which may propagate to fracture. The major cause of heel break is the presence of a micro crack at the heel. This micro crack may be formed during bonding. The causes of micro crack may be attributed to bonding parameters such as high bonding force and excessive ultrasonic energy. The other probable causes are damaged capillary, clogged capillary or over used capillary.

\subsection{Weld Lift}

Weld lifting is the detachment of a wedge bond from the bond pad or bonding post, or the crescent bond from the lead frame bonding finger as shown in figure 5. Weld lift during bond pull test, is an indication of improper process optimization. Similar to ball lift failure, weld lift failures, and even at very high bond pull values is a mark of bad quality and therefore, should not be accepted. The weld lifting could be also caused due to low bond parameters, contamination on lead frame, hard metallization, or improper ultrasonic coupling between the capillary and the lead finger. The cause of this weak coupling between the capillary and the lead finger can be due to lead frame fragility or improper clamping during bonding.

\section{Ball-Bond Shear Test}

The destructive wire bond pull test has limitations, in that it provides very little information on the strength and overall quality of the first bond. A simple wire pull test does not fully explore the characteristics of a ball bond. A quality ball bond can withstand up to ten times the wire pull destruct force but a low quality bond will still take more force to pull off than a wire pull test. Thus pull tests fail in determining the true strength of ball bonds. This factor has led to the development of a complementary wire bond test methodology, the ball-bond shear test.

Ball shear testing is used to assess the integrity of the gold ball-to-bonding pad interface in the gold ball bonding process. It is also a destructive test. Ball shear data reflects the intermetallic formation and its coverage of bonds. It is measured by gram force over the area of the ball formation.

\subsection{Description of the Test}

A shear tool is used to perform this test. During testing this tool pushes the ball bond with a sufficient force. Positioning of the tool is very important during shear testing. The shear ram is positioned just above the bond pad such that the bottom of the shear tool is close to the centre of the ball. The shear tool moves parallel to the bonded surface and shears the ball bond. Figure6 shows the placement of the shear tool.

The improper positioning of the tool is one of most common problem in shear test. During the ball shear test the shearing ram needs to be positioned exactly according to criteria described in the ball shear test specification. For a normally deformed ball, the tool should approach from 2.5 to above the substrate and for large, high balls; it must be no higher than

Otherwise the tool could drag on the substrate. Figure7 shows the step by step movement of Shear Ram and the failure modes of the ball bond.

The failure modes generally observed during a ball shear test are: ball lift / partial metallization lift off, ball shear, bond pad lift and cratering. Let us review and discuss each of the above mentioned failure modes in further detail. 


\subsubsection{Ball lift / Partial Metallization Lift Off}

A ball lift is defined as a separation at the bonding pad interface with little or no intermetallic formation present or remaining. Figure7 (a) shows part of the Al metallization sheared with the ball, leaving partial metallization on the bond pad during shear test.

\subsubsection{Ball Shear (Weld Interface Separation)}

During ball shear test, if the intermetallic formation is pronounced, then the ram may shear the whole ball leaving gold or an intermetallic on the bond pad as shown in the figure7 (b).

\subsubsection{Bond Pad Lift (Substrate Metallization Removal)}

Another failure mode during shear test is bond pad lift. In this failure one can see the separation between the bond pad and the underlying substrate. The aluminum metallization is peeled off leaving exposed. This may occur due to improper adhesion of aluminum metallization. Here the interface between the ball bond and the residual pad attached to the ball remains intact as shown in the figure7(c).

\subsubsection{Cratering}

Cratering is a general term used to describe one type of bonding failure that occurs as the result of mechanical damage to the bond pad or underlying material. This failure occurs on the underside of a wire bond during bond pull or shear test. Cratering occurs as a result of cracks generated under the bond pad during bonding. Figure7 (d) shows cratering effect during ball shear test.

\section{Visual Inspection of Bond Quality}

Inspection of wire bonding had traditionally been done visually, because the human ability to discern faults had been much better than the available machines. This technique is used to ensure the proper ball and wedge bonds. Signs of cracked heels, tearing at the wedge, misplaced wires, inconsistent wire placement, "golf-clubbing" at the gold ball, excessive neck down etc. are visual indications that the wire bonding process is not in control. Visual inspection also verifies whether the bonds are properly placed with respect to the bond pads and bond fingers of the lead frame/substrate. Beside that the visual inspection also screens possible bond defects that may results in open or short, based on a specified defined criterion of wire clearance and close proximity of each bond to the other.

As discussed earlier, bond pull and bond shear test check the quality of bonded interfaces, both on the chip and on the lead finger. However, there are many other failure modes that can only be detected by visual inspection, for example deformed bond, s-ing of wire and probe marks to name a few. Therefore visual inspection is very much essential in today's wire bonding process. Visual inspection of bond quality includes both "Pre-Bonding Inspection" and "Post Bonding Inspection". Pre-Bonding Inspection is necessary for assuring process consistency and reliability. Post Bonding Inspection is necessary to assure package reliability.

\subsection{Pre-Bonding Inspection}

One of the final steps in the manufacture of integrated circuits is the inspection of the probe marks on bonding pads caused during electrical testing of the integrated circuit wafers. Pre-bonding inspection is useful in isolating potential problem areas caused when: the probe mark extends beyond the pad boundary, the mark is too large in size, or extraneous scratch marks have been introduced during electrical testing.

Inspection of the bond pads is facilitated by evaluating parameters which can be used to quantify these defects. These include: size of the pad, location of the pad centre, size of marks on the pad and percentage coverage of pad, location and size of the largest mark.

Other problems on the bonding area that have an impact on the quality of the bond are: discoloration of bond pad (due to corrosion) and photo resist residue. These can be visually inspected and problems resolved. Photo resist residue is not visible under normal lighting. However using polarized UV lighting under a microscope it is possible to identify passivation and photo resist residue problems.

\subsection{Post Bonding Inspection.}

The following list gives a range of problems that one may encounter after the bonding process, and these problems can only be identified visually. The post bonding inspection includes bond quality problems such as: smashed bonds, skinny bonds, misplaced and deformed bonds and wire conditions such as loop height etc.

The visual inspection can be done manually with the assistance of an operator, or with automatic visual inspection systems.

\section{Special Tests for Quality}

The industry uses a variety of techniques for measuring the quality and of bonds. The most frequently used techniques are: visual inspection, bond Pull, bond shear test, bond etching, electrical testing, bake test, thermal cycle/thermal shock 
testing, electro magnetic resonance, surface analysis.

Some of these tests are usable as production monitoring techniques, where as some tests can only be used in the laboratory for evaluation and development of wire bond technology.

\subsection{Bond Etching}

Bond etching measures the condition of layers under the bond pads after bonding. With this technique, the ball (wire) and pad metallization is removed, and the condition of underplayed materials is examined for defects. Wet etching method not only allows detection of subsurface damage caused by equipment parameters, but can also serve as a tool for optimization of the bonding parameters. Typically, $\mathrm{NaOH}$, or $\mathrm{KOH}$ are the chemical solutions of choice used to remove the interconnection material and expose the underlying structure. For example to check if cratering has occurred under the bond pad, we remove the bond pad metallization using potassium hydroxide (KOH) solution.

\subsection{Electrical Testing}

Electrical testing measures the quality of the bond. A non-sticking bond will result in an electrical open, while two adjacent bonds in contact will result in an electrical short. There are special techniques such as "Four Point Probe Testing" to measure the electrical resistance of the Au ball-Al bond pad interface. The resistance increases as the amount of intermetallic compound increases. And an open circuit failure is an indication of extensive voiding at the interface.

\subsection{Bake Test}

Bake test measures the degree of intermetallic compound formation of gold-aluminum (Au/Al). Over diffusion of aluminum atoms into gold can lead to Kirkendall voiding at $\mathrm{Au} / \mathrm{Al}$ interface resulting in bond lifting. By baking the bonded devices at high temperature $\left(175^{\circ}, 240^{\circ}\right.$ or $\left.300^{\circ}\right)$ for different lengths of time, one can determine the long term reliability of the

bond.

\subsection{Surface Analysis}

Surface analysis techniques such as energy-dispersive X-ray analysis (EDX), wave-length dispersive X-ray analysis (WDX), auger, and scanning electron microscopy (SEM) are the failure analysis tools one can use to identify the presence of contamination, extent of intermetallic compound formation, and bond irregularities.

\section{Conclusion}

The quality of a wire bond is determined by the strength of the interfaces between the bond wire and the bond pad as well as the bond wire and the lead finger. In addition, some of the other quality factors that one needs to check for during the wire bonding process are wire sweep, wire sway, wire sagging, heel cracking, bond deformation and many other defects. The quality of the wire bond is controlled by using established methodologies such as bond pull test, bond shear test, visual inspection and special test for quality.

\section{References}

Antony Jiju. (1999). Improving the wire bonding process quality using statistically designed experiments. Microelectronics Journal, 30, 161-168.

Brokelmann Michael, Wallaschek J \& Hesse Hans J. (2005). Model based development of an integrated sensor-actuator system for online quality monitoring in ultrasonic wire bonding, American Society of Mechanical Engineers, 70, 219-226.

Chen J. (2002) Real-time ultrasonic testing of quality of wire bonding. British institute of non-destructive test, 44, 443-445.

Lin T Y, Leonq W S, Chua K H \& Miao Y. (2002). The impact of copper contamination on the quality of the second wire bonding process using X-ray photoelectron spectroscopy method. Microelectronics reliability, 42, 375-380.

LV Lei. (2008). The Process Introduction and quality inspection of wire bonding. Equipment for electronic products manufacturing, 158, 53-60.

Srikanth N. (2005). Effect on ultrasonic bond quality of wire bonds. Proceedings of 7th electronics packaging technology conference, 2, 756-759. 


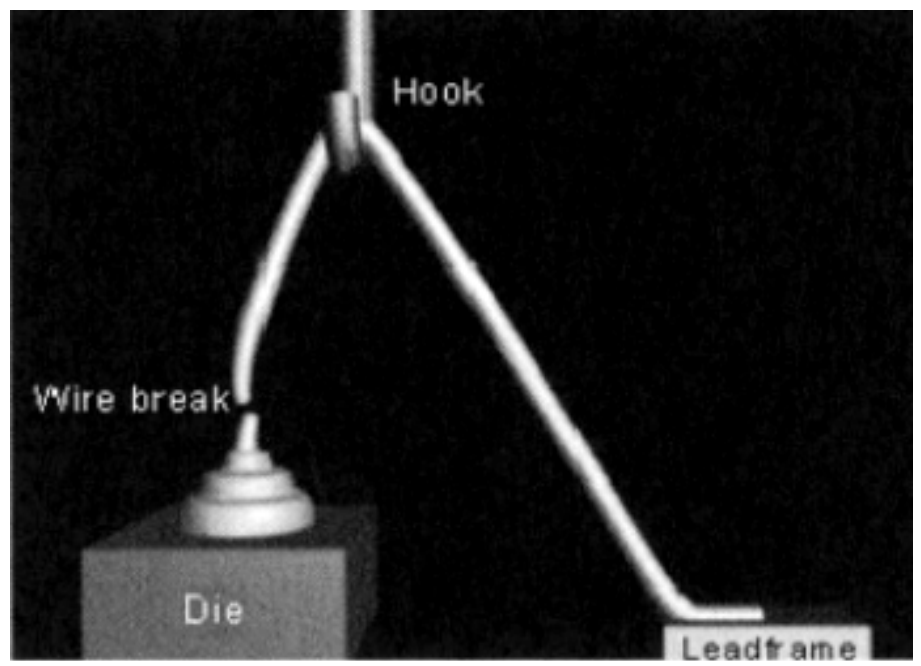

Figure 1. Bond pull test

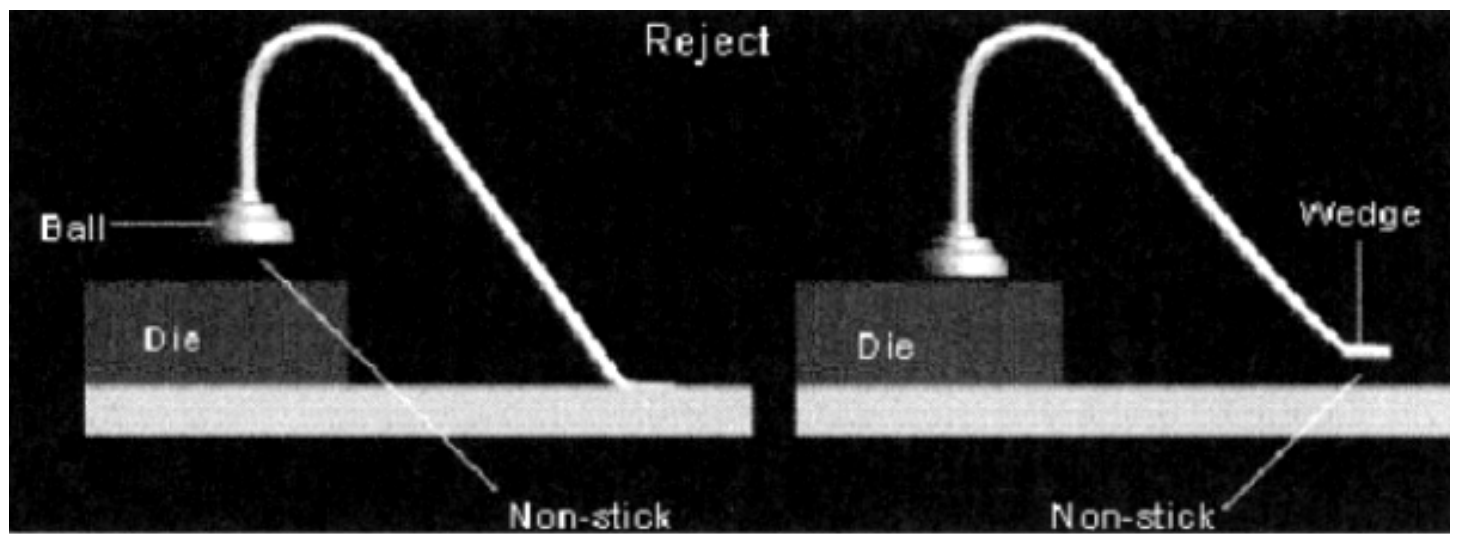

Figure 2. Ball lift and wedge (weld) lift failure

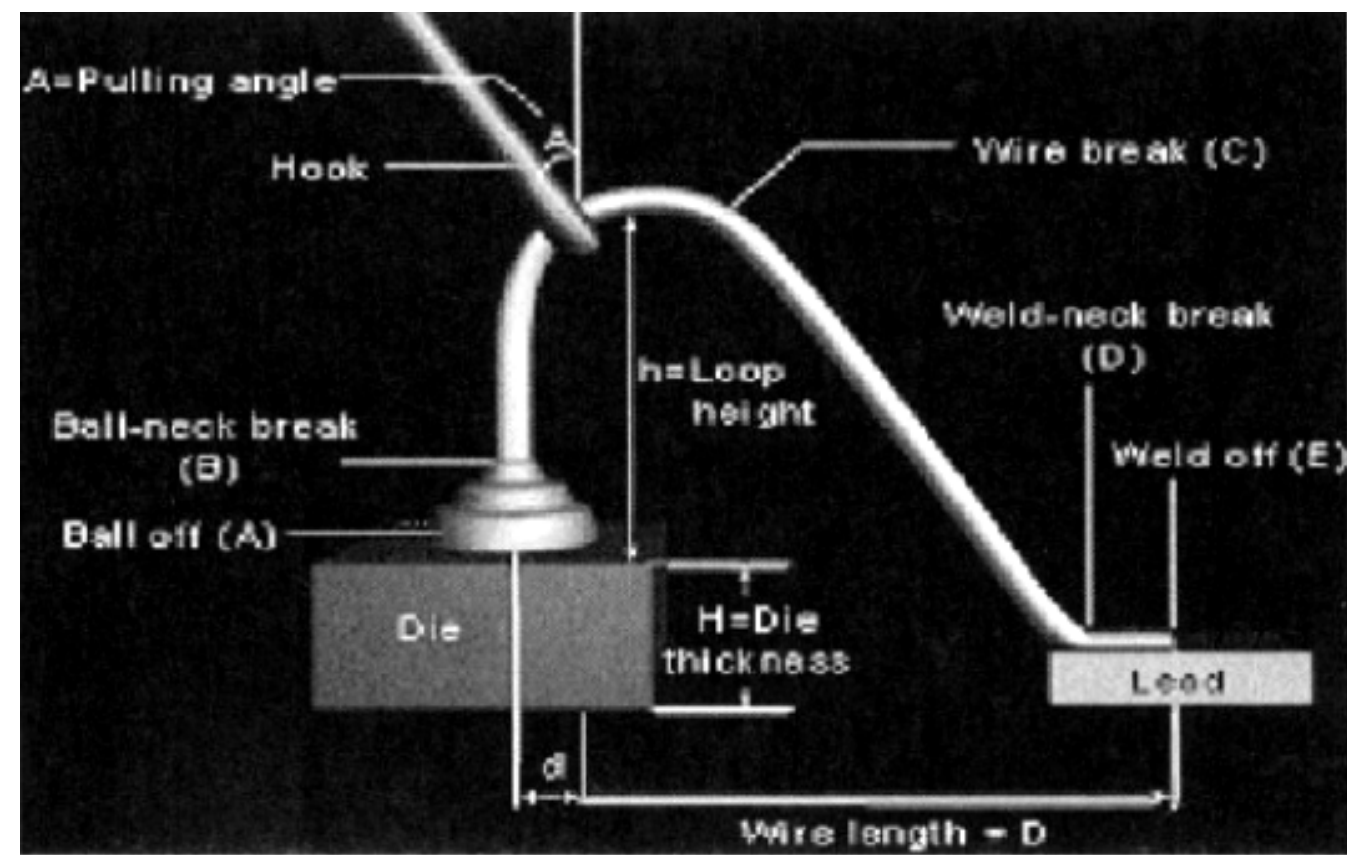

Figure 3. Pull test failure modes 

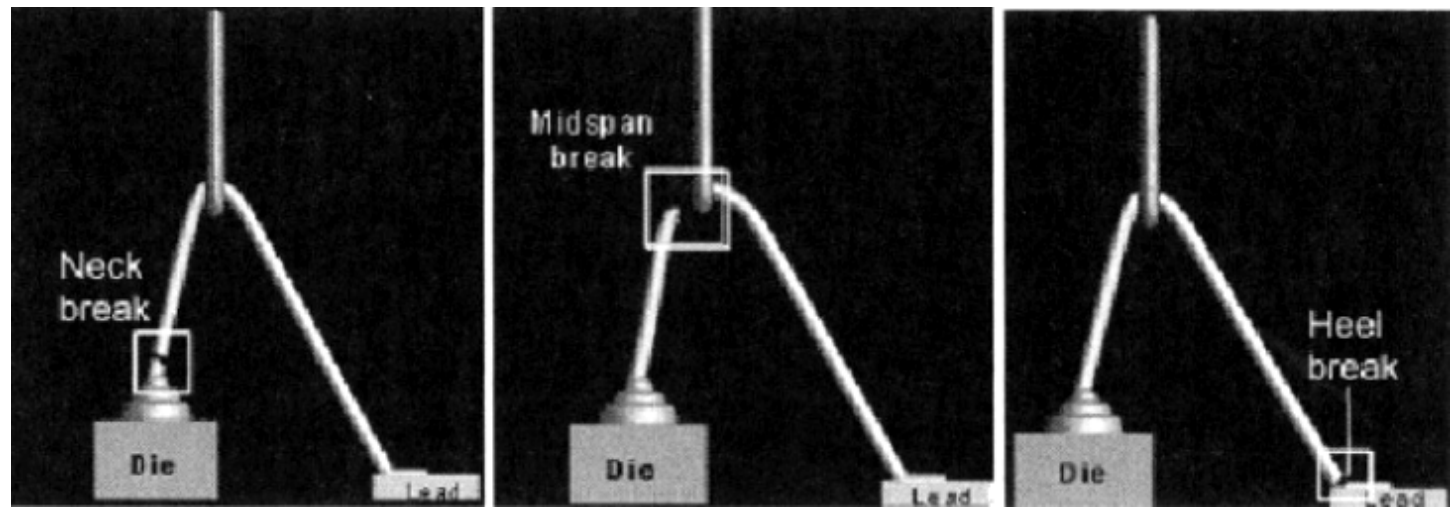

Figure 4. Shows neck break, midspan break and heel break

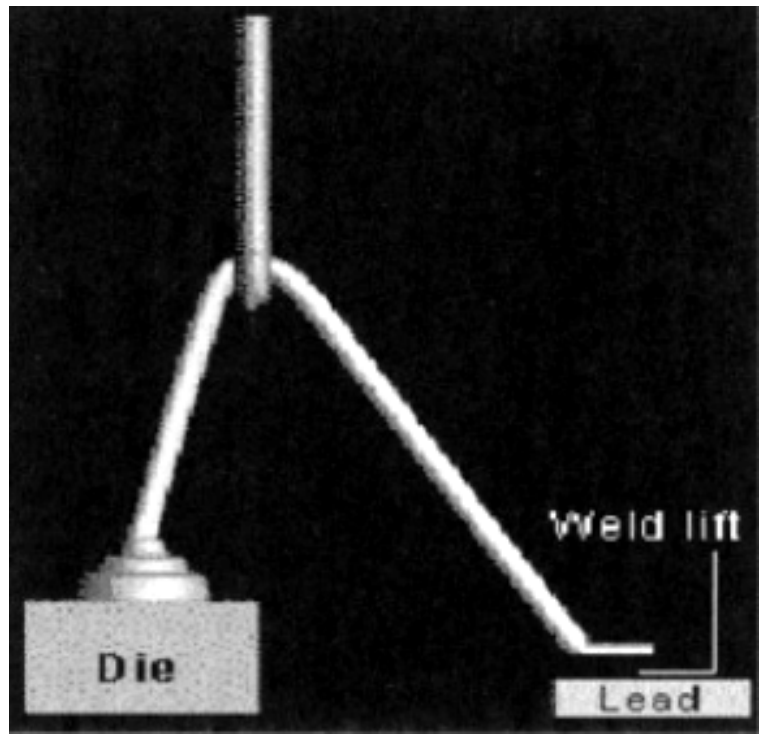

Figure 5. Weld lift

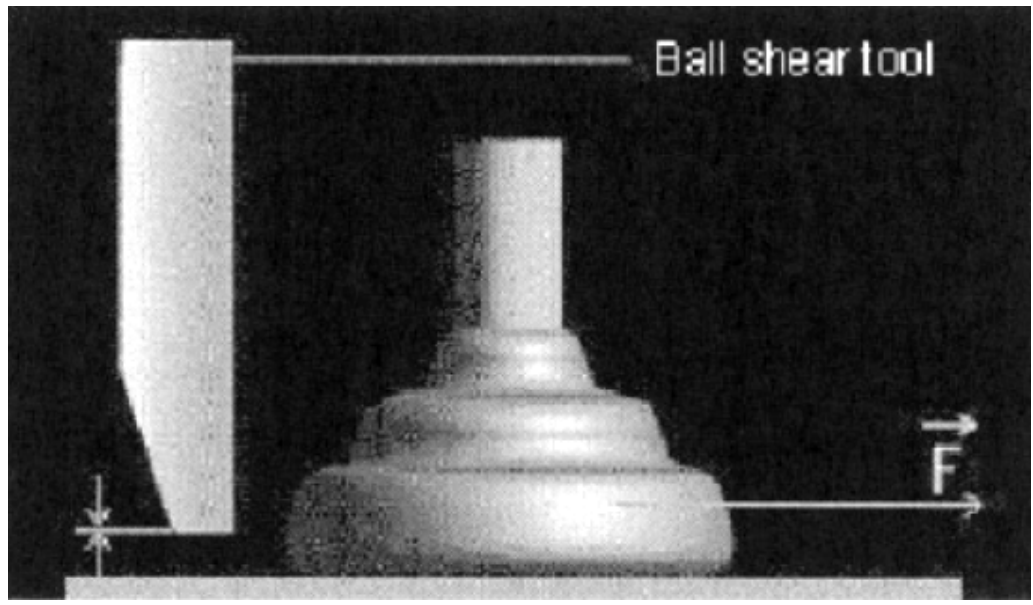

Figure 6. Ball shear test 


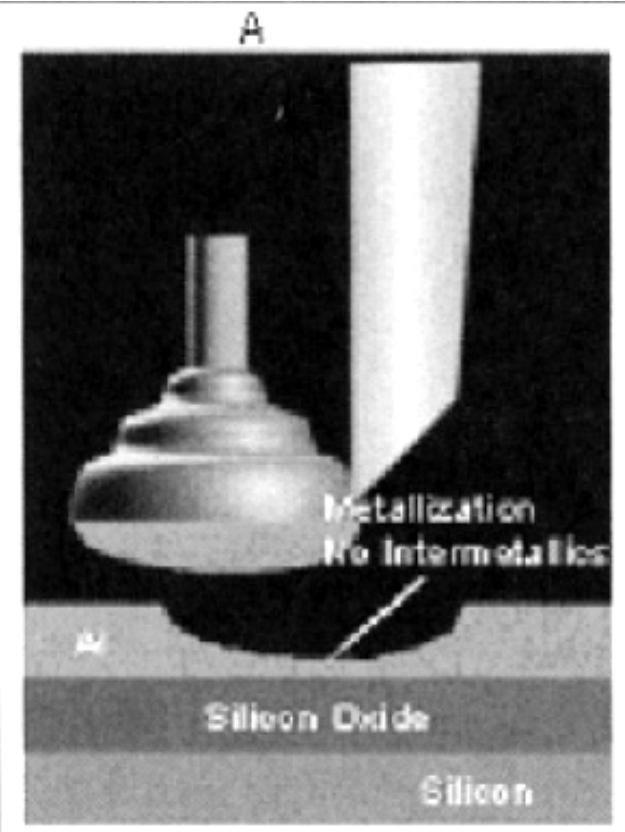

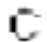

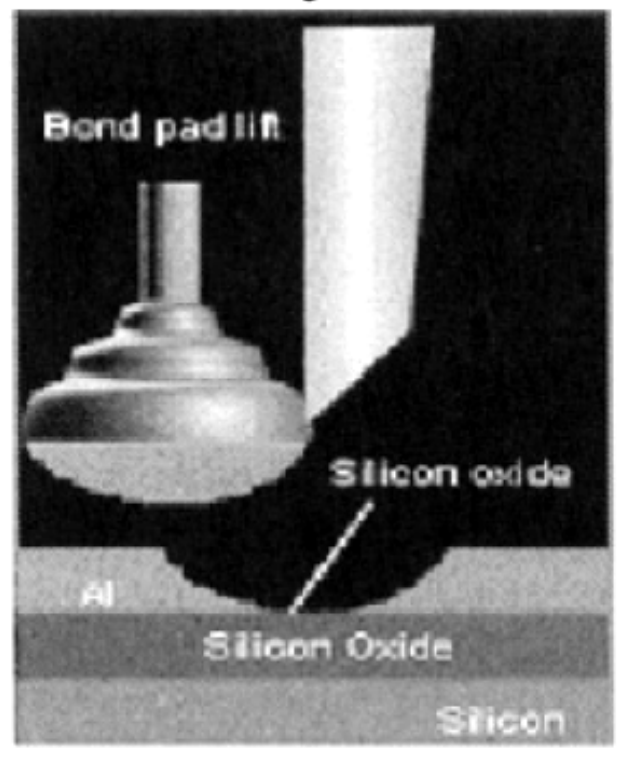

Sricon
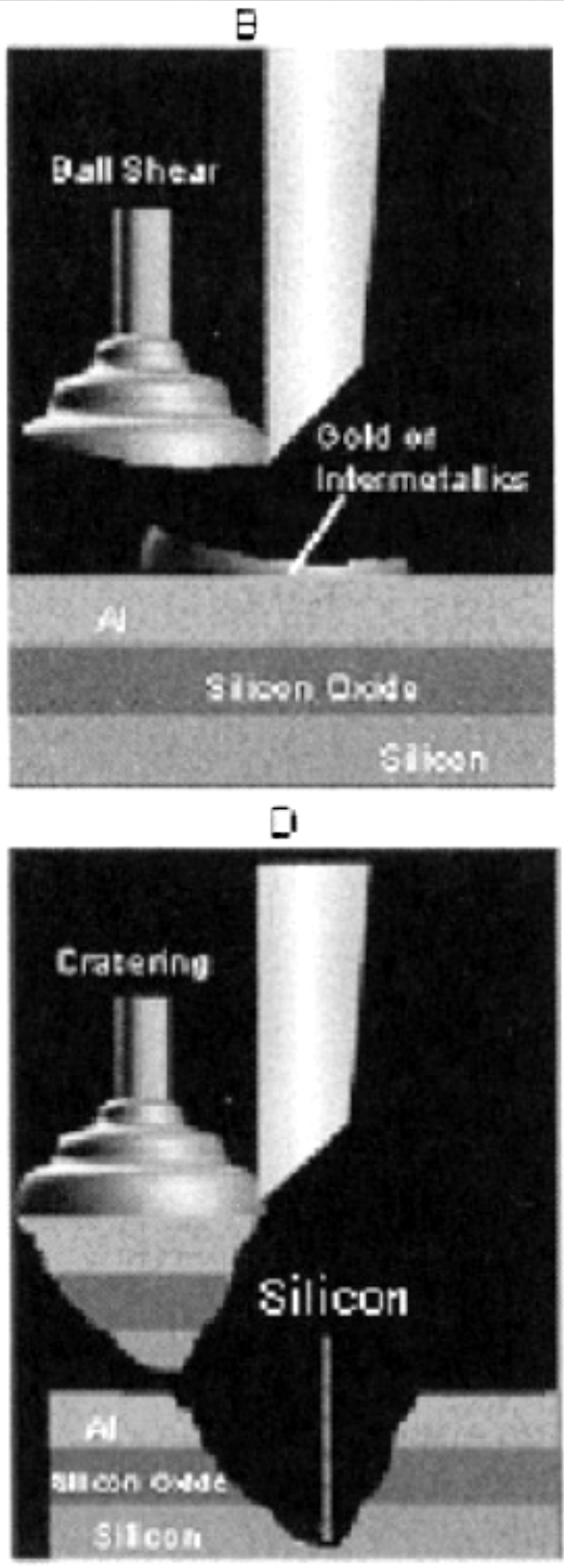

Figure 7. Shear test failure modes (a) Ball lift (b) Ball shear (c) Bond pad lift (d) Cratering 